\title{
Dielectric function of colloidal lead chalcogenide quantum dots obtained by a Kramers-Krönig analysis of the absorbance spectrum
}

\author{
Iwan Moreels, ${ }^{1, *}$ Guy Allan, ${ }^{2}$ Bram De Geyter, ${ }^{1}$ Ludger Wirtz, ${ }^{2}$ Christophe Delerue, ${ }^{2}$ and Zeger Hens ${ }^{1, \dagger}$ \\ ${ }^{1}$ Physics and Chemistry of Nanostructures, Ghent University, BE-9000 Ghent, Belgium \\ ${ }^{2}$ Department ISEN, CNRS-UMR 8520, Institute for Electronics, Microelectronics, and Nanotechnology (IEMN), \\ FR-59652 Villeneuve d'Ascq, France
}

(Received 17 February 2010; revised manuscript received 29 April 2010; published 15 June 2010)

\begin{abstract}
We combined the Maxwell-Garnett effective medium theory with the Kramers-Krönig relations to obtain the complex dielectric function $\epsilon$ of colloidal $\mathrm{PbS}, \mathrm{PbSe}$, and $\mathrm{PbTe}$ quantum dots (Qdots). The method allows extracting both real $\left(\epsilon_{R}\right)$ and imaginary $\left(\epsilon_{I}\right)$ parts of the dielectric function from the experimental absorption spectrum. This enables the quantification of the size-dependent oscillator strength of the optical transitions at different critical points in the Brillouin zone, strongly improving our understanding of quantum confinement effects in these materials. In addition, the static-limit sum rule yields the electronic dielectric constant from the $\epsilon_{I}$ spectrum. Interestingly, values for lead chalcogenide Qdots remain close to the bulk dielectric constant. To verify these trends, we determined the dielectric constant of thin lead chalcogenide layers by ab initio calculations, and the results agree with the experimental data.
\end{abstract}

DOI: 10.1103/PhysRevB.81.235319

PACS number(s): 78.67.Bf, 73.22.-f, 77.22.Ch, 78.67.Hc

\section{INTRODUCTION}

Photonic device development and the optimization of device efficiency is an important aspect of current research on colloidal quantum dots (Qdots). In this respect, the complex dielectric function $\epsilon=\epsilon_{R}+i \cdot \epsilon_{I}$ of colloidal quantum dots (Qdots) is an important materials property. For instance, $\epsilon$ has already been used in the determination of the quantum efficiency of PbSe Qdot solar cells. ${ }^{1}$ With the recent demonstration of colloidal Qdot-based lasing, ${ }^{2,3} \epsilon$ will also become a key parameter in the future design of these lasers, as the Qdot refractive index will contribute to the effective index of the lasing cavity. More generally, understanding the optical properties of a composite material consisting of several Qdot materials and/or sizes will also benefit greatly from the knowledge of $\epsilon$ of their constituents. Fabrication of such structures has already been intensely investigated, for instance, as randomly mixed thin films, ${ }^{4}$ ordered layers of alternating materials, ${ }^{5}$ or as superlattices consisting of two different materials. ${ }^{6,7}$ These composites may find wide spread application. For instance, in the case of a structure composed of different Qdots, energy transfer (ET) from one to the other material is often employed to enhance the luminescence properties of the devices. ${ }^{4,5}$ Improvement of the device efficiency includes optimization of the ET, which depends on the molar extinction coefficient of the acceptor material ${ }^{8}$ and hence on $\epsilon$ due to dielectric confinement. ${ }^{9,10}$ An as yet unexplored potential lies in the application of such composites as negative index metamaterials. Modeling has already shown that the optical properties of such a device strongly depend on the $\epsilon$ of both constituents at frequencies that correspond to transitions close to the band gap. ${ }^{11}$ However, experimental verification is still lacking.

On a more fundamental level, insight in the effects of quantum confinement on the optical properties of Qdots can be improved if $\epsilon$ can be determined over the entire spectral range. Experimental data can then be compared to theory, which is mostly focused on understanding the size depen- dence of the electronic dielectric constant $\epsilon_{0}$. $\epsilon_{0}$ is often described within the generalized Penn Model, ${ }^{12}$ which states that $\epsilon_{0}$ strongly decreases with decreasing size, due to an opening of the band gap for smaller Qdots (quantum confinement). However, recent reports have cast doubt on the validity of the Penn model, proposing that the size dependence arises from a reduction in $\epsilon_{0}$ near the Qdot surface while it remains bulklike in the inner Qdot volume. ${ }^{13,14}$ This implies that $\epsilon_{0}$ merely becomes smaller due to a decreasing Qdot size (increasing the relative surface area).

Considering its importance, surprisingly few experimental reports on $\epsilon$ for colloidal Qdots exist in literature. Data are mainly collected using spectroscopic ellipsometry and results are reported for II-VI [ZnTe, ${ }^{15} \mathrm{CdTe},{ }^{16}$ and HgTe (Ref. 17)], IV-VI [PbSe (Refs. 1 and 18)], and group IV Qdots [Si, ${ }^{19,20}$ Ge (Ref. 21)]. However, a systematic comparison of different materials and Qdot sizes is at present lacking. In this paper, we address this issue by calculating $\epsilon$ for colloidal $\mathrm{PbS}$, $\mathrm{PbSe}$, and PbTe Qdots using a method that we have developed recently. It is based on a Kramers-Krönig (KK) analysis of the Qdot absorption coefficient $\mu$. Our calculation starts from the Qdot absorbance spectrum, which is typically acquired over a spectral range going from UV to wavelengths far beyond the absorption onset. Therefore, we can analyze $\epsilon_{I}$ both around the band gap and at higher lying optical transitions. In addition, the electronic dielectric constant $\epsilon_{0}$ can be derived from $\epsilon_{I}$ by the static-limit sum rule. Results are compared to density-functional calculations, which demonstrate that the generalized Penn model need not be invoked to interpret $\epsilon_{0}$.

\section{DISCRETE KRAMERS-KRÖNIG RELATIONS}

Optical constants of bulk materials can be determined from a KK analysis of the bulk absorption coefficient $\alpha$. The extinction coefficient $k$ is directly related to $\alpha$, and if $k$ can be determined over a wide enough spectral range, the refractive index $n$ can be calculated. From these values, $\epsilon_{R}$ and $\epsilon_{I}$ 
can be determined. However, $\mu$ of colloidal Qdots is given by the Maxwell-Garnett (MG) effective medium theory, ${ }^{22}$ which implies that $\mu$ at a given wavelength $\lambda$ is determined by both real and imaginary parts of $\epsilon\left(n_{s}\right.$ : solvent refractive index)

$$
\mu=\frac{2 \pi}{\lambda n_{s}}\left|f_{L F}\right|^{2} \epsilon_{I}=\frac{2 \pi}{\lambda n_{s}} \frac{9 n_{s}^{4}}{\left(\epsilon_{R}+2 n_{s}^{2}\right)^{2}+\epsilon_{I}^{2}} \epsilon_{I} .
$$

The local field factor $f_{L F}$ clearly influences the Qdot absorption (dielectric confinement ${ }^{9,10}$ ). Although this hampers a straightforward KK analysis, we can still obtain $\epsilon$ by an iterative procedure.

Let us take the following $\mathrm{KK}$ relation as a starting point, which yields $\epsilon_{R}$ at a given frequency $\omega$ from an integration of $\epsilon_{I}$ over the entire frequency domain $(\mathcal{P}$ : Cauchy principal value)

$$
\epsilon_{R}(\omega)=1+\frac{2}{\pi} \mathcal{P} \int_{0}^{\infty} \frac{\omega^{\prime} \epsilon_{I}\left(\omega^{\prime}\right)}{\omega^{\prime 2}-\omega^{2}} d \omega^{\prime} .
$$

It is clear that if, for instance, $\epsilon_{I}$ is known over the entire frequency domain, $\epsilon_{R}$ can be calculated. A practical Qdot absorbance spectrum is, however, typically measured over a wavelength range, not a frequency range, and it is determined at discrete, equidistant wavelengths $\lambda_{k}$, instead of over a continuous range. Using $\omega \cdot \lambda=2 \pi \cdot c$, with $c$ the speed of light, we can transform Eq. (2) into the wavelength domain. In addition, we rewrite the KK relation in a discrete form

$$
\epsilon_{R}\left(\lambda_{j}\right)=1+\frac{2}{\pi_{k \neq j}} \sum_{k} \frac{\lambda_{j}^{2} \Delta \lambda}{\lambda_{k}\left(\lambda_{j}^{2}-\lambda_{k}^{2}\right)} \epsilon_{I}\left(\lambda_{k}\right) .
$$

We take the spacing $\Delta \lambda$ much smaller than the spectral features in the absorbance spectrum, allowing for an adequate sampling of the data. The summation runs from $k=0$ to $\infty$, omitting $k=j$, hereby avoiding infinite values. This is equivalent to using the Cauchy principal value in the continuous KK relation. Considering that $\lambda_{j(k)}=j(k) \cdot \Delta \lambda$, we can simplify this equation

$$
\epsilon_{R, j}=1+\frac{2}{\pi} \sum_{k \neq j} \frac{j^{2}}{k\left(j^{2}-k^{2}\right)} \epsilon_{I, k} .
$$

Finally, we will use a more convenient matrix formalism, writing $\boldsymbol{\epsilon}_{\boldsymbol{R}}$ and $\boldsymbol{\epsilon}_{\boldsymbol{I}}$ as column vectors. In addition, in accordance with the work of Adachi et al., we replace 1 by $\boldsymbol{\epsilon}_{\infty}$ [equal to 1.5 for PbSe (Ref. 23) and 1.7 for $\mathrm{PbS}$ (Ref. 24) and PbTe (Ref. 25)]. This accounts for higher lying transitions not observed in the experimental $\epsilon_{I}$.

$$
\boldsymbol{\epsilon}_{R}=\boldsymbol{\epsilon}_{\infty}+\frac{2}{\pi} \boldsymbol{A} \cdot \boldsymbol{\epsilon}_{I}
$$

with

$$
A_{j, k}=\frac{j^{2}}{k\left(j^{2}-k^{2}\right)}, \quad A_{j, j}=0 .
$$

To demonstrate that the discretization is valid, Fig. 1(a) shows $\epsilon_{I}$ for a virtual material, exhibiting two Lorentz peaks (denoted the TLP particle), plotted on a wavelength scale.
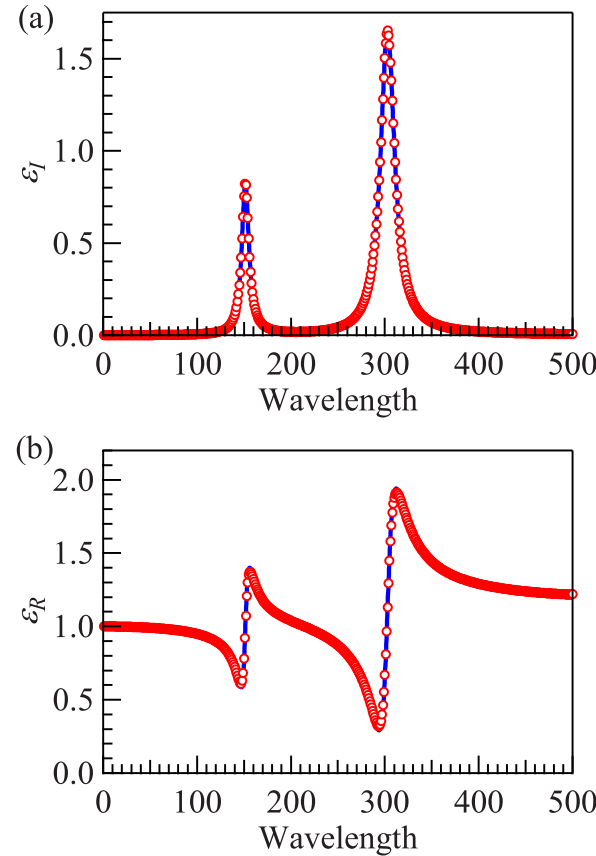

FIG. 1. (Color online) (a) Imaginary part of the dielectric function of the TLP particle. The full line shows the continuous data, the circles represent $\epsilon_{I}$ evaluated at 500 discrete wavelengths. (b) Real part of the dielectric function, calculated from the continuous KK relations $(2$, full line) and the discrete $\mathrm{KK}$ relations $(5, \bigcirc)$. The agreement shows that the discrete $\mathrm{KK}$ relations are valid, even when taking the summation over a finite wavelength interval.

On top of the continuous data, we show the discrete data (500 data points are used). The continuous data are transformed using Eq. (2), the discrete data using Eq. (5), albeit that we now consider a sum over the finite wavelength range shown in Fig. 1. As $\epsilon_{R}$ coincides for both calculations [Fig. 1(b)], we can conclude that the discrete $\mathrm{KK}$ transformation indeed provides an accurate method to calculate $\epsilon_{R}$. In addition, we show that the infinite sum can be replaced by a finite one, at least when extending the wavelength range well into the transparent region $\left(\epsilon_{I}=0\right)$.

\section{CALCULATION OF THE QDOT DIELECTRIC FUNCTION}

\section{A. Method}

Returning to the expression in Eq. (1) for $\mu$ and keeping in mind that this equation is valid at any given $\lambda_{k}$, we see that the calculation of $\epsilon_{I}$ at $N$ given wavelengths requires solving $2 N$ equations [Eqs. (1) and (5)], $N$ of which are quadratic in $\epsilon_{R}$ and $\epsilon_{I}$. Instead of attempting a direct calculation, we develop an iterative approach to solve the problem. Starting from a trial function $\epsilon_{I, 0}$, we calculate $\epsilon_{R, 0}$ using Eq. (5). Both then yield an initial estimate $\mu_{0}$ from Eq. (1) (obviously, $\mu_{0} \neq \mu$ ). We now define $\Delta \epsilon_{I}$ and $\Delta \epsilon_{R}$ as the difference between the trial function and the true values: $\epsilon_{I}$ $=\epsilon_{I, 0}+\Delta \epsilon_{I}, \epsilon_{R}=\epsilon_{R, 0}+\Delta \epsilon_{R}$. After substitution in Eq. (1), a first-order Taylor series expansion yields (using the matrix notation) 


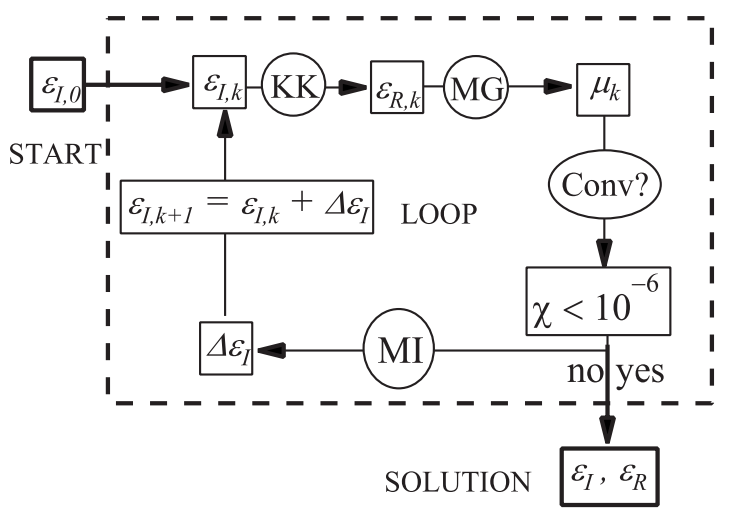

FIG. 2. Schematic representation of the iterative matrix inversion method. KK: Kramers-Krönig transformation, MG: calculation of $\mu$ within the Maxwell-Garnett model, and MI: matrix inversion.

$$
M=C \Delta \epsilon_{I}+D \Delta \epsilon_{R}
$$

with

$$
\begin{gathered}
M_{j}=\frac{\mu}{\mu_{0}}-1, \\
C_{j, j}=\left(\frac{1}{\epsilon_{I, 0}}-\frac{2 \epsilon_{I, 0}}{\left(\epsilon_{R, 0}+2 n_{s}^{2}\right)^{2}+\epsilon_{I, 0}^{2}}\right), \\
D_{j, j}=-\frac{2\left(\epsilon_{R, 0}+2 n_{s}^{2}\right)}{\left(\epsilon_{R, 0}+2 n_{s}^{2}\right)^{2}+\epsilon_{I, 0}^{2}}, \\
C_{j, k}=D_{j, k}=0 .
\end{gathered}
$$

This equation, although only correct up to first order, is linear in $\Delta \epsilon_{I}$ and $\Delta \epsilon_{R}$. Just as for $\epsilon$, the change $\Delta \epsilon$ must obey KK relation: $\Delta \boldsymbol{\epsilon}_{R}=(2 / \pi) \boldsymbol{A} \Delta \boldsymbol{\epsilon}_{I}$ with $\boldsymbol{A}$ already defined in Eq. (5). Hence, combining this equation with Eq. (6), we can calculate $\Delta \boldsymbol{\epsilon}_{\boldsymbol{I}}$ by following matrix inversion:

$$
\Delta \boldsymbol{\epsilon}_{\boldsymbol{I}}=[\boldsymbol{C}+(2 / \pi) \boldsymbol{D} \boldsymbol{A}]^{-1} \boldsymbol{M} .
$$

As already stated, due to the linearization, the corrected trial function $\epsilon_{I, 1}=\epsilon_{I, 0}+\Delta \epsilon_{I}$ does not yet lead to an absorption coefficient $\mu_{1}$ equal to $\mu$. However, by iterating the procedure outlined above, the absorption coefficient calculated at each step $\mu_{k}$ will converge to $\mu$. The iteration is halted once the root-mean-square error $\chi$ is reduced to values below $10^{-6}$. This iterative matrix inversion (IMI) method is summarized in Fig. 2.

To illustrate this approach, we first extract the dielectric function of the TLP particle from its $\mu$ spectrum (Fig. 3). We start from a trial function $\epsilon_{I, 0}$ consisting of three peaks. $\mu$ is calculated from $\epsilon_{I}$ and $\epsilon_{R}$, assuming that the particle is suspended in vacuum (i.e., with a constant refractive index $n_{s}$ $=1$ ). To enable a practical IMI calculation, the infinite wavelength range is replaced by a finite one, extending from $\lambda$ $=0$ well into the transparency region of the material, again using 500 discrete points. As shown in Fig. 3(d), the rootmean-square error $\chi$ rapidly decreases and the IMI procedure already yields the correct dielectric function after only five iterations. Figures 3(e) and 3(f) show the evolution of the
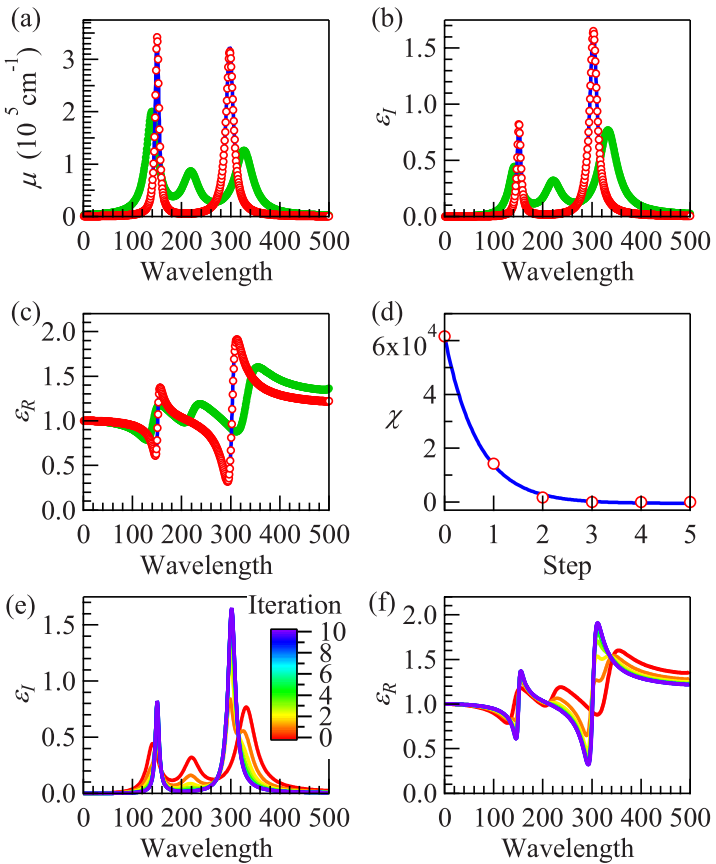

FIG. 3. (Color online) Illustration of the IMI procedure. The full line represents (a) the absorption coefficient, (b) imaginary, and (c) real parts of the dielectric function of the TLP particle. Starting from a trial function $\epsilon_{I, 0}(\bullet)$ exhibiting three peaks, the IMI procedure rapidly yields the correct values for the dielectric function $(\bigcirc)$. (d) Only five iteration steps are needed to bring the error $\chi$ below $10^{-6}$. (e) Imaginary and (f) real parts of the dielectric function for the subsequent steps of the iterative procedure.

dielectric function at different iteration steps. The procedure is slightly modified. In this case, $\Delta \epsilon_{I}$ is divided by two after each step to slow down the convergence and provide a more detailed view of the optimization process. The figure clearly shows how the spurious middle peak is removed and how the left and the right peaks rapidly converge toward the correct value.

\section{B. Results}

First, in order to calculate the Qdot $\epsilon$, we need its $\mu$ spectrum. We start by measuring the absorbance spectrum of a Qdot suspension ( $\mathrm{PbSe}$ Qdots are suspended in $\mathrm{CCl}_{4}, \mathrm{PbS}$, and $\mathrm{PbTe}$ Qdots are suspended in $\mathrm{C}_{2} \mathrm{Cl}_{4}$ ). For PbS (Ref. 26) and $\mathrm{PbSe}$ (Ref. 27) Qdots, we have observed that $\mu$ is bulklike from $3.1 \mathrm{eV}(400 \mathrm{~nm})$ on. Therefore, the Qdot $\mu$ spectrum is obtained by normalizing the experimental Qdot absorbance at $400 \mathrm{~nm}$ to its bulk value, using a known $n_{s} .{ }^{28}$ The respective bulk values used are: $\mu_{\mathrm{PbS}}=1.71 \times 10^{5} \mathrm{~cm}^{-1}$, $\mu_{\mathrm{PbSe}}=2.03 \times 10^{5} \mathrm{~cm}^{-1}$, and $\mu_{\mathrm{PbTe}}=4.49 \times 10^{5} \mathrm{~cm}^{-1}$. Second, the high-energy transitions (in the visible and near UV, the $E 2$ and the $E 3$ transitions along the $\Delta$ direction in the Brillouin zone) in lead chalcogenide Qdots are essentially bulklike, ${ }^{29}$ which already suggests that $\epsilon$ values for the Qdots are identical to bulk values. Hence, we assume that $\epsilon_{I, \mathrm{Qdot}}$ $=\epsilon_{I, \text { bulk }}$ in the range $0-400 \mathrm{~nm}$. In the wavelength range 0-230 nm, where no experimental data are available for $\epsilon_{I, \text { bulk }}$, we use values from the fitted model, as described by 

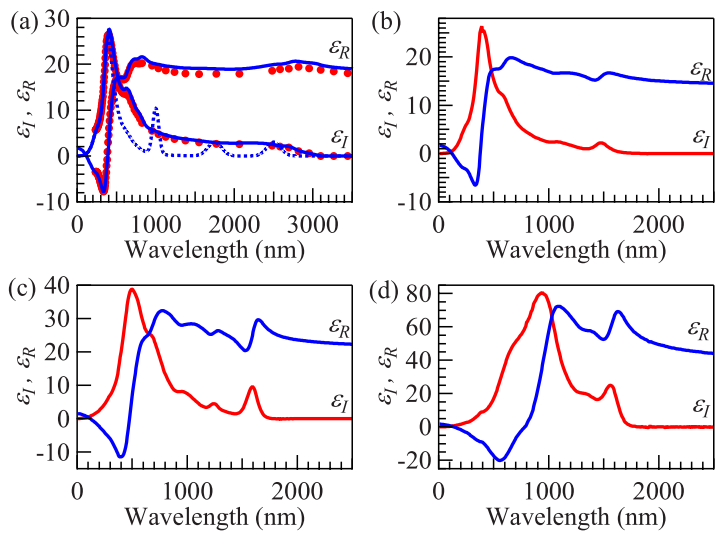

FIG. 4. (Color online) (a) Experimental $\epsilon$ ( $)$ (Ref. 30) and the result of the IMI calculation (full line) for bulk PbS. The initial trial function $\epsilon_{I, 0}$ is indicated by a dotted line. [(b)-(d)] Real and imaginary parts of $\epsilon$ of typical (b) $\mathrm{PbS}$, (c) $\mathrm{PbSe}$, and (d) PbTe Qdots.

Adachi et $a l .^{23-25}$ The equality implies that we can limit the IMI calculation to wavelengths above $400 \mathrm{~nm}$, reducing the calculation time. Finally, for both the IMI calculation and the KK relation, we can set the upper wavelength limit to 3000 $\mathrm{nm}$, well into the transparency region of the Qdot suspensions, as here $\epsilon_{I}$ no longer contributes to $\epsilon_{R}$ [Eq. (5), $\left.\epsilon_{I}=0\right]$.

Before extracting the $\epsilon$ of lead salt Qdots from their respective $\mu$ spectra, we verify the validity of the IMI method on a more realistic system. We start from the $\mu$ spectrum of hypothetical spherical particles with bulk $\mathrm{PbS}$ optical constants. ${ }^{30} \mu$ is determined from Eq. (1), using the $\mathrm{C}_{2} \mathrm{Cl}_{4}$ solvent refractive index ${ }^{28}$ and an interpolation of $\epsilon$ of $\mathrm{PbS}$ (Ref. 30) with $\Delta \lambda=8 \mathrm{~nm}$. Figure 4(a) shows the trial function $\epsilon_{I, 0}$ (dotted line) together with the resulting calculated spectra (full line, calculations are performed as outlined above, in a wavelength range of 400-4000 $\mathrm{nm}$ and using the bulk $\epsilon_{I}$ in the range $0-400 \mathrm{~nm}$ ). Our results agree well with the experimental data (dots). ${ }^{30}$ Note that, as the experimental $\epsilon_{R}$ and $\epsilon_{I}$ are obtained independently from each other, their values are not required to uphold the KK relations while our calculated result must obey the KK relations by definition. An exact match of both data sets is therefore not to be expected, and we can conclude that the IMI method yields an accurate dielectric function.

Despite these results, a possible pitfall of our technique is the potential occurrence of a (nearly) singular matrix, which yields numerical instabilities upon inversion. This might be due to a near zero value of the coefficients $C_{j, j}$ or $D_{j, j}$

$$
\begin{gathered}
C_{j, j}=\frac{1}{\epsilon_{I}}-\frac{2 \epsilon_{I}}{\left(\epsilon_{R}+2 n_{s}^{2}\right)^{2}+\epsilon_{I}^{2}}, \\
D_{j, j}=-\frac{2\left(\epsilon_{R}+2 n_{s}^{2}\right)}{\left(\epsilon_{R}+2 n_{s}^{2}\right)^{2}+\epsilon_{I}^{2}} .
\end{gathered}
$$

We indeed observe that $C_{j, j}$ equals zero when $\epsilon_{I}^{2}=\left(\epsilon_{R}\right.$ $\left.+2 n_{s}^{2}\right)^{2}$ and $D_{j, j}$ equals zero when $\epsilon_{R}=-2 n_{s}^{2}$. Fortunately, for lead chalcogenide materials, this mainly occurs in the wavelength region 0-300 $\mathrm{nm}$. As we use bulk values for $\epsilon_{I}$ in this spectral region, and the IMI calculation is restricted to wave-
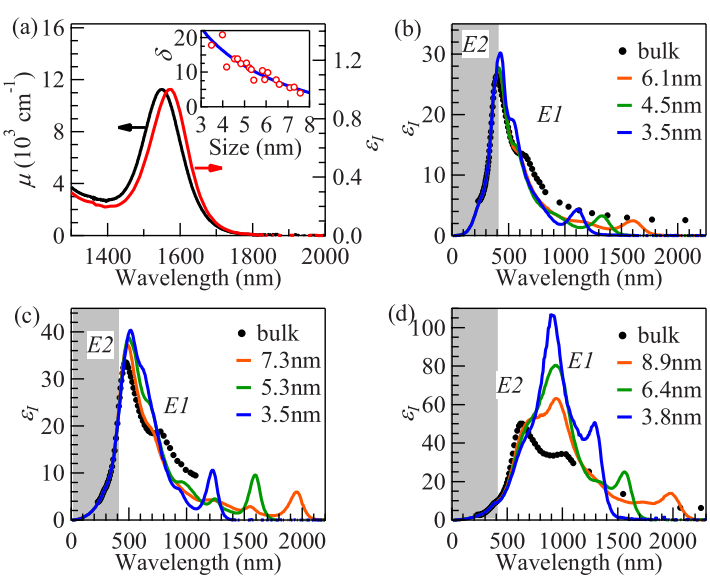

FIG. 5. (Color online) (a) Comparison of $\mu$ and $\epsilon_{I}$ for typical PbSe Qdots. Inset: difference $\delta(\mathrm{meV})$ between the spectral positions of $\mu$ and $\epsilon_{I}$. [(b)-(d)] $\epsilon_{I}$ for three Qdot sizes (full lines) superimposed on bulk data (dots) in the case of (b) PbS, (c) PbSe, (d) and PbTe Qdots. The expansion of Qdot $\epsilon_{I}$ by bulk values $(0-400 \mathrm{~nm})$ is indicated by a gray zone.

lengths above $400 \mathrm{~nm}$, a singular matrix can therefore be avoided. However, to some extent numerical instabilities may persist in our calculations. Therefore, the maximal $\Delta \epsilon_{I}$ value is calculated in each iteration, and if necessary, $\Delta \epsilon_{I}$ is reduced to maintain convergence. This does not hamper the convergence, it merely slows it down.

Proceeding with the determination of the Qdot $\epsilon$, Fig. 4 shows the results of an IMI calculation for typical lead chalcogenide Qdots (typical meaning here that the sizes range from 3 to $10 \mathrm{~nm}$ and the size dispersion is below 10\%). All $\epsilon$ spectra are calculated starting from a trial function equal to the $\epsilon_{I}$ of their respective bulk material. ${ }^{30}$ Clearly, in contrast with the absorbance spectrum, where optical transitions in the visible are cloaked by a strongly rising $f_{L F},{ }^{29}$ all optical transitions are readily distinguished in the spectra of $\epsilon_{I}$. In addition, $\epsilon_{R}$ spectra now also provide further insight into the Qdot optical properties. We observe strong antiresonances around the exciton transitions, demonstrating a strong modulation of $\epsilon_{R}$ due to the sharp Qdot absorption peaks. Comparing the PbSe Qdot spectrum to recently published data using ellipsometry, ${ }^{1}$ we observe an improved signal-to-noise ratio around the band gap. This demonstrates that the IMI method is a powerful technique, enabling the quantification of the optical properties of colloidal Qdot suspensions and thin films over the entire spectral range.

Figure 5(a) compares $\epsilon_{I}$ with $\mu$ at the band gap of $\mathrm{PbSe}$ Qdots. We clearly observe a slight (4-21 meV) redshift of the band-gap transition for the $\epsilon_{I}$ spectrum, which is due to the removal of $f_{L F}$. This agrees with pseudopotential calculations on InAs Qdots, ${ }^{31}$ and consequently, for an in-depth comparison between experiment and theory, either $f_{L F}$ should be included in the calculations ${ }^{14}$ or removed from experimental data. However, given the small shift observed, in practical calculations this effect might be neglected.

Figures 5(b)-5(d) show the full $\epsilon_{I}$ spectrum of three typical Qdots of varying size $d$ (full lines) superimposed on their respective bulk spectrum (dots). ${ }^{23-25}$ First, from the $\epsilon_{I}$ spectra, we can calculate the oscillator strength $f_{\text {if }}$ per particle of the band-gap transition ( $f$ sum rule) 

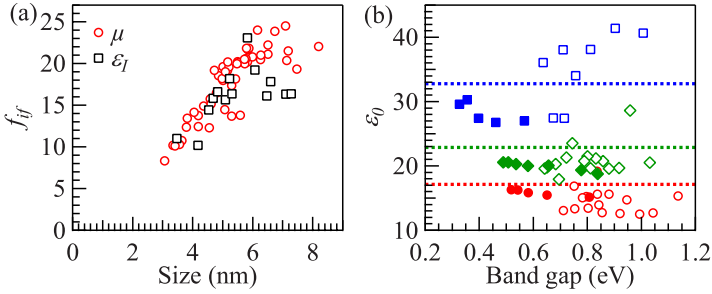

FIG. 6. (Color online) (a) $f_{i f}$, calculated from $\epsilon_{I}(\square)$, agrees well with previous calculations starting from $\mu(\bigcirc)$. (b) We observe no size dependence for the experimental values of $\epsilon_{0}$ shown by open symbols for $\mathrm{PbS}(\bigcirc), \mathrm{PbSe}(\diamond)$, and $\mathrm{PbTe}(\square)$ Qdots. Experimental results agree with DFT calculations on lead chalcogenide quantum wells (closed symbols). Furthermore, the average Qdot value is close to the respective bulk value (Ref. 34) (dotted line).

$$
f_{i f}=\frac{2 \epsilon_{0} m_{e}}{\pi e^{2}} \frac{\pi d^{3}}{6} \int_{1 \mathrm{~S} 1 \mathrm{~S}} \omega \epsilon_{I}(\omega) d \omega .
$$

We integrate $\epsilon_{I}$ over the first absorption peak. Rewritten in a discrete version and assuming a small peak width, this yields

$$
f_{i f}=\frac{\epsilon_{0} m_{e}}{3 \hbar^{2}} d^{3} E_{0}^{2} \sum_{k} \frac{\epsilon_{I}}{k} .
$$

Practically, in accordance with a calculation of $f_{\text {if }}$ from the absorbance spectrum, ${ }^{26,27}$ we take the sum over the long wavelength half of the peak, and multiply the result by two. For PbSe Qdots, $f_{i f}$ calculated from $\epsilon_{I}$ is compared with previously published values ${ }^{27}$ in Fig. 6(a). The agreement between both data sets again demonstrates that the IMI method provides accurate data and proves that our previous assumption, that $f_{\text {if }}$ can be calculated using the bulk $\epsilon_{R}$, holds for lead chalcogenide Qdots.

Focusing now on the $E 1$ and the $E 2$ transitions in the visible spectral region, we observe that the oscillator strength and spectral position of the $E 2$ transition show no significant confinement effects. The Qdot E2 resonances are comparable to bulk for all three materials. This was already suggested by the bulklike nature of $\mu$ in this spectral region. A slight increase and redshift is observed for the smaller PbSe Qdots [Fig. 5(b)], but this might be due to the merging of the $E 1$ and the $E 2$ transitions. In contrast, we observe strong quantum confinement effects in all three lead chalcogenide Qdots for the $E 1$ transition. In the case of $\mathrm{PbS}$ Qdots, the transition shifts to shorter wavelengths with decreasing Qdot size. In the case of PbSe Qdots, the blueshift is accompanied by an increase in the oscillator strength of the $E 1$ transition with respect to the E2 transition. This effect is even more pronounced for PbTe Qdots. The results presented here confirm previous experimental and theoretical data on $\epsilon$ of $2 \mathrm{D} \mathrm{PbSe}$ Qdots, electrodeposited on gold surfaces, where a blueshift and relative increase in the $E 1$ transition with decreasing Qdot height has also been observed, ${ }^{18}$ and recently published results of Koole et al., ${ }^{32}$ who reported a blueshift of the $E 1$ transition. The origin of the different behavior of the $E 1$ and the $E 2$ transitions can be found in the lead chalcogenide band structure: $E 1$ and $E 2$ arise from optical transitions at two different $\boldsymbol{k}$ points in the Brillouin zone, at saddle points characterized by different positive or negative effective masses. ${ }^{33}$

\section{Dielectric constant}

The electronic dielectric constant $\epsilon_{0}$ is defined as the real part of $\epsilon$ at energies far below the band-gap transition. It can be calculated from $\epsilon_{I}$ using the static-limit sum rule (discrete version)

$$
\epsilon_{0}=\epsilon_{\infty}+\frac{2}{\pi} \sum_{k=1}^{N} \frac{\epsilon_{I}}{k} .
$$

Figure 6(b) shows the results for $\mathrm{PbS}, \mathrm{PbSe}$, and $\mathrm{PbTe}$ Qdots, plotted as a function of their respective band-gap energy (open symbols). Interestingly, we observe no significant size dependence. Averaged over all samples, we find: $\epsilon_{0, \mathrm{PbS}}$ $=14.5 \pm 1.8, \epsilon_{0, \mathrm{PbSe}}=21.0 \pm 2.5$, and $\epsilon_{0, \mathrm{PbTe}}=35.4 \pm 5.4$. The average values are close to the respective bulk values of $17.15(\mathrm{PbS}), 22.85$ (PbSe), and 32.8 (PbTe). ${ }^{34}$ These results are somewhat surprising. Comparable experimental data are scarce, ${ }^{20}$ but theoretical studies on $\epsilon_{0}$ of silicon Qdots show that $\epsilon_{0}$ decreases with decreasing particle size, either due to the opening of the band gap (generalized Penn model) ${ }^{12}$ or by a strong decrease in $\epsilon_{0}$ near the Qdot surface (rendering the averaged $\epsilon_{0}$ size dependent). ${ }^{13,14}$ In contrast, for lead chalcogenide Qdots we find values in reasonable agreement with bulk data.

In order to study the effect of quantum confinement on the dielectric constant of lead chalcogenides, we performed $a b$ initio calculations on slabs in the (001) orientation. The thickness ranges from three layers to 15 layers. The slabs are organized in a periodic supercell, the size of which is such that adjacent slabs are separated by a vacuum with a width of 12 atomic layers. To calculate the electronic ground state of the slabs, we use density-functional theory (DFT) in the generalized-gradient approximation as implemented in the code QUANTUM ESPRESSO. ${ }^{35}$ Wave functions are expanded in plane waves with an energy cutoff at $40 \mathrm{Ry}$ and the first Brillouin zone is sampled by a $16 \times 16 \times 1 k$-point mesh. The effect of core electrons (including the $5 d$ electrons of lead) is represented by Vanderbilt ultrasoft pseudopotentials. For the calculation of the dielectric constant, $\epsilon_{0}$, we follow a strategy recently employed by Nakamura et al. ${ }^{36}$ for ultrathin $\mathrm{Si}$ films. Starting point is the calculation of the total electrostatic potential of the slab, averaged over the parallel directions, in the presence of a homogeneous external electric field perpendicular to the slab [Fig. 7(a)]. This field is simulated by a saw-tooth potential, where the kinks in the slope fall in the vacuum region far away from the slab [dashed line in Fig. $7(\mathrm{~b})]$. Since the applied electric field $\left(10^{-4}\right.$ a.u. $)$ is relatively weak, the distortion of the total potential is hardly visible in Fig. 7(a). However, if we regard the antisymmetric part of the total potential, $\bar{v}_{t o t}(z)=\left[v_{t o t}(z)-v_{t o t}(-z)\right] / 2$, the effect of the electric field is clearly observed [Fig. 7(b)]: the saw-tooth potential polarizes the slab, resulting in a reduction in the electric field (i.e., the slope of the potential) $E^{\text {int }}$ in the interior of the slab, and an enhancement of the exterior field $E^{\text {ext }}$. The average dielectric constant is calculated as the ratio of external and (average) internal fields 

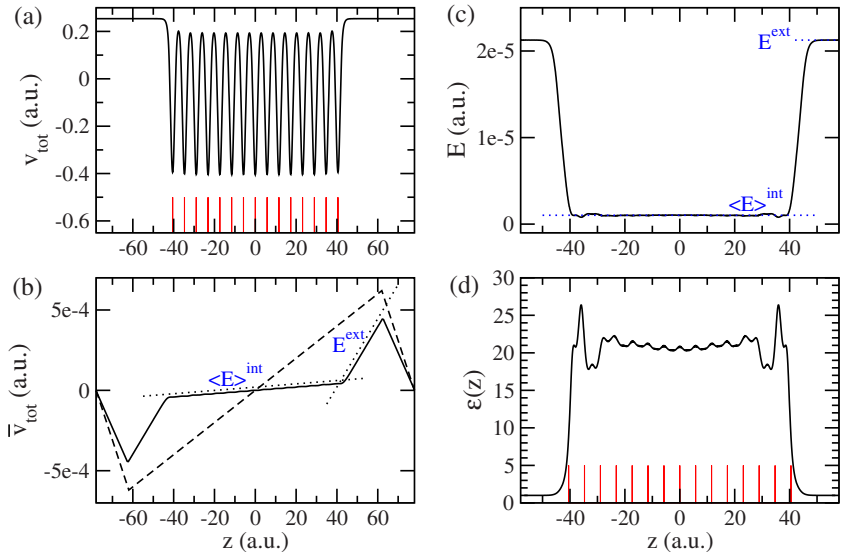

FIG. 7. (Color online) Calculation of the local dielectric constant, $\epsilon(z)$, of a PbSe slab with 15 layers. (a) Total potential, averaged over the $x-y$ plane. (b) Antisymmetric part of the total potential (solid line), induced by the saw-tooth potential (dashed line). (c) Local electric field. (d) Local dielectric constant (averaged over the $x-y$ plane). Vertical bars in panels (a) and (d) mark the positions of the atomic planes.

$$
\epsilon_{0}=\frac{E^{\text {ext }}}{\left\langle E^{\text {int }}\right\rangle} .
$$

It is also instructive to regard the variation in the field on a local scale: $E^{\text {int }}(z)=\partial \bar{v}_{t o t} / \partial z$ is displayed in Fig. $7(\mathrm{c})$. In order to smooth out effects of subatomic variations in the electron density, we have averaged the electric field over the width of an atomic plane. The "local dielectric constant," $\epsilon(z)=E^{\text {ext }} / E^{\text {int }}$, is displayed in Fig. 7(d). Similar to the case of silicon slabs, ${ }^{13,36} \epsilon(z)$ converges quickly to an almost constant value within four layers from the surface, which confirms that the opening of the band gap induced by the confinement does not influence the dielectric response. ${ }^{13}$ The enhancement of the local dielectric constant at the surface layers (present in all the calculations of slabs of different width) is probably due to some surface states. Furthermore, we note that for slabs with more than nine layers, $\epsilon_{0}$ is close to the value $\epsilon(z)$ in the middle of the slab.

In an alternative calculation of the dielectric function of the slabs, we employed density-functional perturbation theory ${ }^{37}$ as implemented in QUANTUM ESPRESSO. We use the same slab geometries with $n_{s}=3, \ldots, 15$ layers as described above. Here, a separation by $n_{v}=8$ layers of vacuum is sufficient. The code yields the average dielectric function, $\bar{\epsilon}$ of the unit cell, which has a component parallel to the slab layers, $\overline{\boldsymbol{\epsilon}}_{\|}$, and perpendicular to the slab layers, $\overline{\boldsymbol{\epsilon}}_{\perp}$. We define the parallel component of the dielectric function in the slab, $\epsilon_{\|}$, in such a way that the effective medium value of the dielectric function coincides with the average dielectric function

$$
\bar{\epsilon}_{\|}=\left(n_{s} \epsilon_{\|}+n_{v} \epsilon_{\mathrm{vac}}\right) /\left(n_{s}+n_{v}\right) .
$$

Similarly, the perpendicular component $\epsilon_{\perp}$ is defined by

$$
\frac{n_{s}+n_{v}}{\bar{\epsilon}_{\perp}}=\frac{n_{s}}{\epsilon_{\perp}}+\frac{n_{v}}{\epsilon_{\mathrm{vac}}} .
$$

The dielectric function of vacuum $\epsilon_{\mathrm{vac}}$ is equal to one.

The results of the calculation are shown in Table I. We observe that both the parallel and the perpendicular components of the dielectric function in the slab converge very quickly toward a value slightly above the calculated ${ }^{38}$ bulk value of $\epsilon_{\mathrm{bulk}}=21.03$. The fact that $\epsilon_{0}$ in the layers is larger than the bulk value is due to the strong enhancement of the local dielectric function at the surface [Fig. 7(d)] and has not been observed for silicon slabs. ${ }^{13,14,36}$ This difference may be explained by the efficient passivation of the silicon surfaces by hydrogen ${ }^{13,14,36}$ while, for lead chalcogenide materials, there are surface states resonant in the bands ${ }^{33}$ which can induce local enhancement of the dielectric response.

Our calculations have been performed on slabs. However, since the effect of quantum confinement on both the parallel and the perpendicular components of $\epsilon_{0}$ is basically absent, we expect our result to hold as well for quantum wires and quantum dots where DFT calculations become quickly unfeasible. For this reason, we compare in Fig. 6(b) the values measured on quantum dots (open symbols) with the calculations on slabs (closed symbols). With increasing band gap, the theoretical $\epsilon_{0}$ slightly decreases, yet remains close to the respective bulk value. For $\mathrm{PbS}$ and $\mathrm{PbSe} \mathrm{Qdots}$, the calculated $\epsilon_{0}$ agrees well with experimental data. For PbTe, the calculations systematically underestimate $\epsilon_{0}$ due to shortcomings in the description of the electronic bands. ${ }^{39}$ Nevertheless, for the materials studied here our DFT calculations

TABLE I. DFT band gaps and dielectric constants, calculated with DFPT and with the electric-field

\begin{tabular}{|c|c|c|c|c|c|c|}
\hline$n_{s}$ & $\begin{array}{c}E_{g} \\
(\mathrm{eV})\end{array}$ & $\bar{\epsilon}_{\|}$ & $\bar{\epsilon}_{\perp}$ & $\epsilon_{\|}$ & $\epsilon_{\perp}$ & $\epsilon_{\perp}^{\text {field }}$ \\
\hline 3 & 0.838 & 6.77 & 1.35 & 22.15 & 23.59 & 18.77 \\
\hline 5 & 0.778 & 8.91 & 1.58 & 21.56 & 21.97 & 19.37 \\
\hline 7 & 0.656 & 10.52 & 1.80 & 21.39 & 21.50 & 20.03 \\
\hline 9 & 0.580 & 11.76 & 2.02 & 21.32 & 21.36 & 20.00 \\
\hline 11 & 0.536 & 12.74 & 2.23 & 21.28 & 21.34 & 20.26 \\
\hline 13 & 0.508 & 13.55 & 2.44 & 21.27 & 21.29 & 20.56 \\
\hline 15 & 0.489 & 14.20 & 2.64 & 21.24 & 21.23 & 20.56 \\
\hline
\end{tabular}
methods (last column) for different PbSe slabs; $n_{s}$ denotes the number of layers in the slab. Equations (14) and (15) are used to convert the parallel/perpendicular components of $\bar{\epsilon}$ to the ones of $\epsilon$. 
clearly show that $\epsilon_{0}$ is hardly influenced by the opening of the band gap. Quantum confinement leads to an increase in the minimum energy for electron-hole pairs and thus one might think that it should lead to a reduced dielectric constant. However, the average energy for electron-hole pair transitions remains almost constant down to very thin slabs and thus also the dielectric constant remains almost unchanged. ${ }^{13}$

\section{CONCLUSIONS}

The results presented here provide a major step forward in the understanding of the Qdot optical properties, as until now, it has been difficult to access $\epsilon$ experimentally, especially at energies around the band gap. We have shown here that the IMI method enables the determination of $\epsilon$ over the entire spectral range of interest, enabling a detailed study of both its real and imaginary parts. In addition, the static-limit sum rule yields the dielectric constant from the $\epsilon_{I}$ spectrum. Experimental values agree with DFT calculations, showing that $\epsilon_{0}$ is essentially bulklike. From a practical perspective, we provide much needed experimental data on colloidal lead salt Qdots, useful for optimizing photonic devices. Finally, as the IMI method can be applied to any suspension for which the MG model is valid, we present a powerful calculation of the optical properties of a material from a measurement of the absorbance spectrum.

\section{ACKNOWLEDGMENTS}

I.M. is a researcher with the FWO-Vlaanderen. This project is funded by the Belgian Science Policy Office (Grant No. IAP P6/10), the EU Seventh Framework Program (EUFP7 ITN Herodot), and the FWO-Vlaanderen (Grant No. G.0.144.08.N.10). Calculations have been performed at the computing center of Lille University and at the IDRIS supercomputing center, Orsay (Project No. 091827). *iwan.moreels@ugent.be

‘zeger.hens@ugent.be

${ }^{1}$ M. Law, M. C. Beard, S. Choi, J. M. Luther, M. C. Hanna, and A. J. Nozik, Nano Lett. 8, 3904 (2008).

${ }^{2}$ Y. Chan, J. S. Steckel, P. T. Snee, J. M. Caruge, J. M. Hodgkiss, D. G. Nocera, and M. G. Bawendi, Appl. Phys. Lett. 86, 073102 (2005).

${ }^{3}$ S. Hoogland, V. Sukhovatkin, I. Howard, S. Cauchi, L. Levina, and E. H. Sargent, Opt. Express 14, 3273 (2006).

${ }^{4}$ S. Nizamoglu, O. Akin, and H. V. Demir, Appl. Phys. Lett. 94, 243107 (2009).

${ }^{5}$ T. Franzl, T. A. Klar, S. Schietinger, A. L. Rogach, and J. Feldmann, Nano Lett. 4, 1599 (2004).

${ }^{6}$ E. V. Shevchenko, D. V. Talapin, N. A. Kotov, S. O'Brien, and C. B. Murray, Nature (London) 439, 55 (2006).

${ }^{7}$ K. Overgaag, W. Evers, B. de Nijs, R. Koole, J. Meeldijk, and D. Vanmaekelbergh, J. Am. Chem. Soc. 130, 7833 (2008).

${ }^{8}$ C. R. Kagan, C. B. Murray, M. Nirmal, and M. G. Bawendi, Phys. Rev. Lett. 76, 1517 (1996).

${ }^{9}$ T. Takagahara, Phys. Rev. B 47, 4569 (1993).

${ }^{10}$ D. Ricard, M. Ghanassi, and M. C. Schanneklein, Opt. Commun. 108, 311 (1994).

${ }^{11}$ Y. Fu, L. Thylen, and H. Agren, Nano Lett. 8, 1551 (2008).

${ }^{12}$ A. C. Sharma, J. Appl. Phys. 100, 084301 (2006).

${ }^{13}$ C. Delerue, M. Lannoo, and G. Allan, Phys. Rev. B 68, 115411 (2003).

${ }^{14}$ F. Trani, D. Ninno, and G. Iadonisi, Phys. Rev. B 76, 085326 (2007).

${ }^{15}$ F. Ahmed, A. E. Naciri, J. J. Grob, M. Stchakovsky, and L. Johann, Nanotechnology 20, 305702 (2009).

${ }^{16}$ P. Babu Dayal, B. R. Mehta, and P. D. Paulson, Phys. Rev. B 72, 115413 (2005).

${ }^{17}$ V. Rinnerbauer, K. Hingerl, M. Kovalenko, and W. Heiss, Appl. Phys. Lett. 89, 193114 (2006).

${ }^{18}$ Z. Hens, D. Vanmaekelbergh, E. S. Kooij, H. Wormeester, G. Allan, and C. Delerue, Phys. Rev. Lett. 92, 026808 (2004).
${ }^{19}$ L. Ding, T. P. Chen, Y. Liu, C. Y. Ng, and S. Fung, Phys. Rev. B 72, 125419 (2005).

${ }^{20}$ H. G. Yoo and P. M. Fauchet, Phys. Rev. B 77, 115355 (2008).

${ }^{21}$ M. Mansour, A. E. Naciri, L. Johann, S. Duguay, J. J. Grob, M. Stchakovsky, and C. Eypert, J. Phys. Chem. Solids 67, 1291 (2006).

${ }^{22}$ A. Sihvola, J. Electromagn. Waves Appl. 15, 715 (2001).

${ }^{23}$ N. Suzuki, K. Sawai, and S. Adachi, J. Appl. Phys. 77, 1249 (1995).

${ }^{24}$ H. Kanazawa and S. Adachi, J. Appl. Phys. 83, 5997 (1998).

${ }^{25}$ N. Suzuki and S. Adachi, Jpn. J. Appl. Phys., Part 1 33, 193 (1994).

${ }^{26}$ I. Moreels et al., ACS Nano 3, 3023 (2009).

${ }^{27}$ I. Moreels, K. Lambert, D. De Muynck, F. Vanhaecke, D. Poelman, J. C. Martins, G. Allan, and Z. Hens, Chem. Mater. 19, 6101 (2007).

${ }^{28}$ H. H. Marvin, Phys. Rev. 34, 161 (1912).

${ }^{29}$ I. Moreels and Z. Hens, Small 4, 1866 (2008).

${ }^{30} \mathrm{~S}$. Adachi, Optical Properties of Crystalline and Amorphous Semiconductors (Kluwer Academic, Boston, 1999).

${ }^{31}$ A. Franceschetti, A. Williamson, and A. Zunger, J. Phys. Chem. B 104, 3398 (2000).

${ }^{32}$ R. Koole, G. Allan, C. Delerue, A. Meijerink, D. Vanmaekelbergh, and A. J. Houtepen, Small 4, 127 (2008).

${ }^{33}$ G. Allan and C. Delerue, Phys. Rev. B 70, 245321 (2004).

${ }^{34}$ J. N. Zemel, J. D. Jensen, and R. B. Schoolar, Phys. Rev. 140, A330 (1965).

${ }^{35} \mathrm{~S}$. Baroni et al., http://www.quantumespresso.org/

${ }^{36}$ J. Nakamura, S. Ishihara, A. Natori, T. Shimizu, and K. Natori, J. Appl. Phys. 99, 054309 (2006).

${ }^{37}$ S. Baroni, S. de Gironcoli, A. Dal Corso, and P. Giannozzi, Rev. Mod. Phys. 73, 515 (2001).

${ }^{38}$ This value was obtained using a $20 \times 20 \times 20$ Monkhorst-Pack sampling. Note that it depends very sensititvely on the employed pseudopotentials and the lattice constant (Ref. 39).

${ }^{39}$ O. Kilian, G. Allan, and L. Wirtz, Phys. Rev. B 80, 245208 (2009). 